\title{
Time paradox in Quantum Gravity
}

\author{
Alfredo Macías and Hernando Quevedo

\begin{abstract}
The aim of this work is to review the concepts of time in quantum mechanics and general relativity to show their incompatibility. We show that the absolute character of Newtonian time is present in quantum mechanics and also partially in quantum field theories which consider the Minkowski metric as the background spacetime. We discuss the problems which this nondynamical concept of time causes in general relativity that is characterized by a dynamical spacetime.
\end{abstract}

Mathematics Subject Classification (2000). Primary 46N50; Secondary 85A99.

Keywords. Quantum Gravity, Time problem, Quantization methods.

\section{Introduction}

Our present description of the laws of physics may be characterized as obtained from two types of constituents. The first type of constituent are theoretical frameworks which apply to all physical phenomena at any instant. These "universal" or "frame" theories are Quantum Theory, i.e., all matter is of microscopic origin, Special and General Relativity, i.e., all kinds of matter locally have to obey the principles of Lorentz symmetry and behave in the same way in gravitational fields, and Statistical Mechanics which is a method to deal with all kinds of systems for a large number of degrees of freedom. The second type of constituent is nonuniversal and pertains to the description of the four presently-known interactions: the electromagnetic, the weak, the strong, and the gravitational. The first three interactions are all described within a single formalism, in terms of a gauge theory. So far only gravity has not been successfully included into that scheme. One reason for that might be that gravity appears on both sides: it is an interaction but it is at the same time also a universal theory. Universal theories like relativity and gravity are geometric in origin and do not rely on the particular physical system under consideration, whereas a description in terms of a particular interaction heavily makes use of the particular particle content. Therefore, gravity plays 
a distinguished role which may be the reason for the difficulty encountered in attempting to unify the other interactions with gravity and attempting to quantize gravity [1, 2].

The concepts of time in quantum mechanics and general relativity are drastically different from each other.

One one hand, time in quantum mechanics is a Newtonian time, i.e., an absolute time. In fact, the two main methods of quantization, namely, canonical quantization method due to Dirac and Feynman's path integral method are based on classical constraints which become operators annihilating the physical states, and on the sum over all possible classical trajectories, respectively. Therefore, both quantization methods rely on the Newton global and absolute time. The absolute character of time in quantum mechanics results crucial for its interpretation, i.e., matrix elements are evaluated at fixed time, and the internal product is unitary, i.e., conserved in time, and it implies conservation of the total probability. Therefore, time is part of the classical background, which is needed for the interpretation of measurements. Moreover, the introduction of a time operator in quantum mechanics is thus problematic. The time parameter appears explicitly in the Schrödinger equation, together with the imaginary unit. Since time is absolute can be factorized, for instance, in the canonical quantization, reducing the quantization problem to the construction of a Hilbert space for stationary states.

The transition to (special) relativistic quantum field theories can be realized by replacing the unique absolute Newtonian time by a set of timelike parameters associated to the naturally distinguished family of relativistic inertial frames. In this manner, the time continues to be treated as a background parameter.

On the other hand, time in general relativity is dynamical and local. Hence, it is not an absolute time. The geometry of spacetime influences material clocks in order to allow them to display proper time, and the clocks react on the metric changing the geometry. Therefore, the metric itself results to be a clock, and the quantization of the metric can be understood as a quantization of time [3].

The above mentioned quantization methods, when applied to general relativity lead to the Wheeler-DeWitt equation [4. It is well known that, as a second order functional differential equation, the Wheeler-DeWitt equation presents familiar problems when one tries to turn the space of its solutions into a Hilbert space [5].

In full, general relativity does not seem to possess a natural time variable, while quantum theory relies quite heavily on a preferred time. Since the nature of time in quantum gravity is not yet clear, the classical constraints of general relativity do not contain any time parameter, and one speaks of the time paradox.

The aim of the present work is to review the concepts of time in both quantum mechanics and general relativity. Our understanding of time is in the context of the canonical quantization approach to quantum mechanics and quantum field theory. This is why we review in section 2 the axioms of canonical quantization, emphasizing the role of time at each step. Then, in section 3 we discuss the role of the time parameter in general relativity and establish its dynamical character. 
Sections 4 and 5 are devoted to brief descriptions of how time enters the problem of canonical quantization on minisuperspaces and midisuperspaces, respectively. Section [ [ contains a discussion on the main approaches used to attack the problem of time. Finally, section $\mathbf{7}$ is devoted to the conclusions.

\section{Time in canonical quantization}

Quantum theory is based on a certain procedure of quantization of a classical system which consists of a series of axioms. The standard and most used procedure is canonical quantization, whose starting point is the Hamiltonian describing the classical system. It is interesting that, like any other physical theory, there is no proof for quantum theory. The only thing we know for sure is that the experimental observations of Nature do not contradict the predictions of quantum theory, at least within the range of measurements accessible to current experimental devices. In canonical quantization time plays a very important role in all the axioms which are postulated as the fundamentals of this method. First, the mere fact that one needs to know the Hamiltonian of the system implies that a certain time parameter has to be chosen in order to define the variables in phase space. To be more specific let us briefly recall the main axioms of canonical quantization.

In the case of quantum mechanics for a system with only bosonic degrees of freedom these axioms can be stated as follows:

I) There exists a Hilbert space $\mathbf{H}$ for the quantum system and the elements of $\mathbf{H}$ are the quantum states $|\psi\rangle$ of the system. The Hilbert space is supposed to be equipped with an inner product, i.e. a positive definite Hermitian norm on $\mathbf{H}$. Often the inner product of two elements $|\phi\rangle$ and $|\psi\rangle$ of $\mathbf{H}$ is denoted as $\langle\phi \mid \psi\rangle$.

II) A classical observable $A$ is replaced by a Hermitian operator $\hat{A}$ acting on elements of $\mathbf{H}$. When the observable $A$ is measured, the result must coincide with one of the eigenvalues of $\hat{A}$. It is also assumed that for any physical state $|\psi\rangle \in \mathbf{H}$, there exists an operator for which the state $|\psi\rangle$ is one of its eigenstates.

III) If $q_{i}$ and $p_{j}(i, j=1,2, \ldots n=$ number of bosonic degrees of freedom of the system), are the variables in phase space $\mathbf{R}^{2 n}$, the corresponding operators must obey the commutation relations at a fixed time $t$

$$
\left[\hat{q}_{i}, \hat{q}_{j}\right]=0, \quad\left[\hat{p}_{i}, \hat{p}_{j}\right]=0, \quad\left[\hat{q}_{i}, \hat{p}_{j}\right]=i \delta_{i j},
$$

where we are using units with $\hbar=1$. This axiom can be generalized to include the case of phase spaces other than $\mathbf{R}^{2 n}$ (see, for instance, 6, 7, 8]).

IV) If $\hat{A}$ does not depend explicitly on time, its evolution in time is determined by Heisenberg's evolution equation:

$$
\frac{d \hat{A}}{d t}=\frac{1}{i}[\hat{A}, \hat{H}] .
$$

The formal solution of this equation $\hat{A}(t)=e^{i \hat{H} t} \hat{A}(0) e^{-i \hat{H} t}$ can be used to obtain the equivalent Schrödinger picture in which the operators $\hat{A}(0)$ are timeindependent and instead the states become time-dependent through the unitary 
transformation $|\psi(t)\rangle=e^{-i \hat{H} t}|\psi\rangle$. Then, the evolution of a state of the physical system turns out to be determined by the Schrödinger equation

$$
i \frac{d}{d t}|\psi(t)\rangle=\hat{H}|\psi(t)\rangle \text {. }
$$

V) In general, the observation of $A$ in a physical system at a fixed time $t$ yields random results whose expectation value is given by

$$
\langle A\rangle_{t}=\frac{\langle\psi|\hat{A}(t)| \psi\rangle}{\langle\psi \mid \psi\rangle} .
$$

These are the axioms that lie on the basis of canonical quantization for classical systems with a finite number of degrees of freedom. The time parameter $t$ plays a very important role in determining the phase space, i.e. the choice of canonical positions $q_{i}$ and momenta $p_{i}$. Fortunately, the time used in classical mechanics is the absolute Newtonian time which is defined up to constant linear transformations. Thus, the conjugate momenta are determined up to a multiplicative constant which does not affect the main structure of the phase space. This absolute time is then used with no changes in the quantization scheme described in the above axioms.

Time enters explicitly in axioms III and V, since the commutation relations must be satisfied at a given moment in time and the results of any observation lead to expectation values which are well-defined only if time is fixed. This crucial role of time can be rephrased in terms of the wave function. Indeed, if we define the wave function $\psi(t, x)$ as $\psi(t, x)=\langle x \mid \psi(t)\rangle$, fixing its normalization, means that it must be normalized to one at a fixed time.

The equation of evolution (2.3) represents changing relations amongst the fundamental entities (operators) of this construction. This equation indicates which operator has to be used to describe the physical system at a given time. When time changes, Heisenberg's equation explains which operator in Hilbert space corresponds to the new state of the physical system.

These observations indicate that in canonical quantization time is an "external" parameter. It is not a fundamental element of the scheme, but it must be introduced from outside as an absolute parameter which coincides with the Newtonian time. Since there is no operator which could be associated with time, it is not an observable.

The transition to quantum field theory is performed in a straightforward manner, although many technical details have to be taken into account [9]. The main variables are now the value of the field $\varphi(\mathbf{x})$ at each spatial point and the conjugate momentum $\pi(\mathbf{x})$ for that particular value. The collection of all the values of the field, together with the values of the conjugate momenta, represents the variables of the new phase space. Axioms I - V are then postulated for the corresponding phase space variables. Some changes are necessary in order to consider the new "relativistic" time. In particular, the commutation relation

$$
[\hat{\varphi}(x), \hat{\varphi}(y)]=0
$$


is valid for any spacetime points $x$ and $y$ which are spacelike separated. The main difference in the treatment of fields is that the time parameter is that of special relativity. Instead of the absolute Newtonian time, we now have a different parameter associated to each member of the distinguished class of inertial frames. The two absolute concepts of Newtonian physics, i.e. space and time, are now replaced by the single concept of spacetime. Nevertheless, in special relativity spacetime retains much of the Newtonian scheme. Although it is not possible to find an absolute difference between space and time, spacetime is still an element of the quantum theory which does not interact with the field under consideration. That is to say, spacetime remains as a background entity on which one describes the classical (relativistic) and quantum behavior of the field.

In other words, one could say that an observer with the ability to "see" only the physical characteristics of spacetime cannot determine if he/she is "living" on a spacetime with a classical or a quantum field. Spacetime in quantum field theory is therefore an external entity like the absolute external time in quantum mechanics. The dynamics of the field does not affect the properties of spacetime which is therefore a nondynamical element of the theory.

\section{Time in general relativity}

To implement the canonical quantization procedure in general relativity one needs to find the classical Hamiltonian. As mentioned above, such a formulation requires an explicit choice of time or, equivalently, a slicing of spacetime into spatial hypersurfaces associated to the preferred chosen time. This is the Arnowitt-DeserMisner (ADM) [10] approach which splits spacetime into space and time. The pseudo-Riemannian manifold describing the gravitational field is therefore topologically equivalent to $\mathbf{R} \times \Sigma_{t}$, where $\mathbf{R}$ represents the "time axis", and $\Sigma_{t}$ are constant-time hypersurfaces, each equipped with a set of three coordinates $\left\{x^{i}\right\}$ and a non-degenerate 3-metric $q_{i j}$. The relationship between the local geometry on $\Sigma_{t}$ and the 4 -geometry can be recovered by choosing an arbitrary point on $\Sigma_{t}$ with coordinates $x^{i}$ and displacing it by an infinitesimal amount $d t$ normal to $\Sigma_{t}$. The result of this infinitesimal displacement induces an infinitesimal change in proper time $\tau$, which can be written as $d \tau=N d t$, where $N=N\left(x^{\mu}\right)$ is the lapse function, and an infinitesimal change in spatial coordinates, which can be written as $x^{i}(t+d t)=x^{i}(t)-N^{i} d t$, where $N^{i}=N^{i}\left(x^{\mu}\right)$ is the shift vector. Then the 4 -dimensional interval connecting the starting $x^{i}$ and ending $x^{i}+d x^{i}$ points of this infinitesimal displacement is given by the ADM-metric

$$
d s^{2}=-N^{2} d t^{2}+q_{i j}\left(d x^{i}+N^{i} d t\right)\left(d x^{j}+N^{j} d t\right) .
$$

Notice that this splitting of spacetime explicitly depends on the choice of the time parameter $t$. Indeed, the tensorial quantities $N, N^{i}$, and $q_{i j}$ can be given different values by means of a general diffeomorphism. 
The Einstein-Hilbert action on a manifold $M$ with vanishing cosmological constant reads

$$
S_{E H}=\frac{1}{16 \pi G} \int L d^{4} x=\frac{1}{16 \pi G} \int_{M} \sqrt{-g} R d^{4} x \pm \frac{1}{8 \pi G} \int_{\partial M} \sqrt{q} K d^{3} x,
$$

in terms of (3.1) it becomes a function of the intrinsic metric $q_{i j}$ and its derivatives of first order in time. The boundary term in (3.2) is necessary in the variation to cancel terms that arise after integrating by parts 11]. It is positive (negative) in case of spacelike (timelike) components of $\partial M$ and vanishes when the manifold is spatially compact. The phase space is then constructed by means of the configuration space variables $q_{i j}$ and their canonically conjugate momenta $\pi^{i j}=\partial L / \partial\left(\partial_{t} q_{i j}\right)$ which are related to the extrinsic curvature of the 3-dimensional hypersurface $\Sigma_{t}$ as embedded in the 4-dimensional spacetime. The resulting Hamiltonian turns out to be that of a constrained system, indicating that the phase space variables are not independent. A straightforward calculation shows that the Einstein-Hilbert action can be written as (dropping boundary terms)

$$
S_{E H}=\int d t \int_{\Sigma} d^{3} x\left(\pi^{i j} \partial_{t} q_{i j}-N H_{\perp}-N^{i} H_{i}\right) .
$$

Since this action does not contain time derivatives of $N$ and $N^{i}$, their variation leads to the Hamiltonian constraint (super-Hamiltonian constraint)

$$
H_{\perp}:=16 \pi G G_{i j k l} \pi^{i j} \pi^{k l}-\frac{1}{16 \pi G} \sqrt{q}^{(3)} R=0,
$$

and the constraint of spatial diffeomorphisms (super-momentum constraint)

$$
H^{i}(\mathbf{x})=-2^{(3)} \nabla_{j} \pi^{i j}=0 .
$$

Here $q$ is the determinant and ${ }^{(3)} R$ the curvature scalar of the $3-$ metric $q_{i j}$. The covariant derivative with respect to $q_{i j}$ is denoted by ${ }^{(3)} \nabla_{j}$. The DeWitt supermetric is defined as

$$
G_{i j k l}:=\frac{1}{2 \sqrt{q}}\left(q_{i k} q_{j l}+q_{j k} q_{i l}-q_{i j} q_{k l}\right) .
$$

Einstein's field equations are now the standard Hamilton equations for the corresponding action with the Poisson brackets defined according to

$$
\left\{q_{i j}(\mathbf{x}), \pi^{k l}\left(\mathbf{x}^{\prime}\right)\right\}=\delta_{(i}^{k} \delta_{j}^{l} \delta\left(\mathbf{x}, \mathbf{x}^{\prime}\right) .
$$

This special slicing, in which the structure of the spatial hypersurfaces $\Sigma_{t}$ is determined as the $t=$ const. surfaces, leads to the first computational complication for the algebra of diffeomorphisms. In fact, the diffeomorphism invariance in the starting 4-dimensional spacetime is well defined in terms of the corresponding Lie group. When this spacetime diffeomorphism invariance is projected along and normal to the spacelike hypersurfaces $\Sigma_{t}$, one obtains

$$
\begin{gathered}
\left\{H_{i}(\mathbf{x}), H_{j}\left(\mathbf{x}^{\prime}\right)\right\}=H_{i}\left(\mathbf{x}^{\prime}\right) \partial_{j}^{\mathbf{x}} \delta\left(\mathbf{x}, \mathbf{x}^{\prime}\right)-H_{j}(\mathbf{x}) \partial_{i}^{\mathbf{x}^{\prime}} \delta\left(\mathbf{x}, \mathbf{x}^{\prime}\right) \\
\left\{H_{i}(\mathbf{x}), H_{\perp}\left(\mathbf{x}^{\prime}\right)\right\}=H_{\perp}(\mathbf{x}) \partial_{i}^{\mathbf{x}} \delta\left(\mathbf{x}, \mathbf{x}^{\prime}\right)
\end{gathered}
$$




$$
\left\{H_{\perp}(\mathbf{x}), H_{\perp}\left(\mathbf{x}^{\prime}\right)\right\}=q^{i j}(\mathbf{x}) H_{i}(\mathbf{x}) \partial_{j}^{\mathbf{x}^{\prime}} \delta\left(\mathbf{x}, \mathbf{x}^{\prime}\right)-q^{i j}\left(\mathbf{x}^{\prime}\right) H_{i}\left(\mathbf{x}^{\prime}\right) \partial_{j}^{\mathbf{x}} \delta\left(\mathbf{x}, \mathbf{x}^{\prime}\right)
$$

The fact that the right-hand side of Eq. (3.10) contains the 3-metric explicitly implies that the projected algebra of constraints is not a Lie algebra. This is a consequence of the choice of time which leads to considerable computational complications for quantization [12. One could try to choose a specific gauge in accordance to the invariance associated with the algebra (3.8)-(3.10), then solve the constraints (3.4) and (3.5), and finally quantize the resulting system with the "true" degrees of freedom. It turns out that in general the final equations are tractable only perturbatively, and lead to ultraviolet divergences (for further details see, for example, [13, 14]).

An alternative approach consists in applying the canonical quantization procedure to the complete collection of variables in phase space. The variables $q_{i j}$ and $\pi^{j k}$ are declared as operators $\hat{q}_{i j}$ and $\hat{\pi}^{j k}$ which are defined on the hypersurface $\Sigma_{t}$ and satisfy the commutation relations

$$
\begin{aligned}
& {\left[\hat{q}_{i j}(\mathbf{x}), \hat{q}_{k l}\left(\mathbf{x}^{\prime}\right)\right]=0,} \\
& {\left[\hat{\pi}^{i j}(\mathbf{x}), \hat{\pi}^{k l}\left(\mathbf{x}^{\prime}\right)\right]=0,} \\
& {\left[\hat{q}_{i j}(\mathbf{x}), \hat{\pi}^{k l}\left(\mathbf{x}^{\prime}\right)\right]=i \delta_{i}^{k} \delta_{j}^{l} \delta\left(\mathbf{x}, \mathbf{x}^{\prime}\right) .}
\end{aligned}
$$

According to Dirac's quantization approach for constrained systems, the operator constraints must annihilate the physical state vectors, i.e.,

$$
\begin{gathered}
\hat{H}_{\perp} \Psi[q]=0, \\
\hat{H}_{i} \Psi[q]=0,
\end{gathered}
$$

at all points in $\Sigma_{t}$. If the standard representation

$$
\hat{q}_{i j} \Psi[q]:=q_{i j} \Psi[q], \quad \hat{\pi}^{i j} \Psi[q]:=-i \frac{\delta \Psi[q]}{\delta q_{i j}},
$$

is used, the constraint $\hat{H}_{i} \Psi[q]=0$ requires that $\Psi[q]$ behaves as a constant under changes of the metric $q_{i j}$ induced by infinitesimal diffeomorphisms of the 3dimensional hypersurface $\Sigma_{t}$. In this specific representation the Hamiltonian constraint (3.12) becomes the Wheeler-DeWitt equation

$$
-16 \pi G G_{i j k l} \frac{\delta^{2} \Psi[g]}{\delta q_{i j} \delta q_{k l}}-\frac{1}{16 \pi G}{ }^{(3)} R \Psi[g]=0 .
$$

In canonical quantization this is considered as the main dynamical equation of the theory, since classically the function(al) $H_{\perp}$ is associated with the generator of displacements in time-like directions. That is to say, $H_{\perp}$ is the generator of the classical evolution in time. By analogy with quantum mechanics or quantum field theory one expects that the Wheeler-DeWitt equation (3.15) determines the evolution among quantum states. Unfortunately, Eq.3.15 makes no reference to 
time, i.e., all the quantities entering it are defined on the 3-dimensional hypersurface $\Sigma_{t}$. This is one of the most obvious manifestations of the problem of time in general relativity. The situation could not be worse! We have a quantum theory in which the main dynamical equation can be solved without considering the evolution in time.

Some researchers interpreted this result as an indication of the necessity of a completely different "timeless" approach to quantum theory 15, 16, 17. This approach is still under construction and although, in principle, some conceptual problems can be solved some other problems related to "time ordering" and "time arbitrariness" appear which are, at best, as difficult as the above described problems of time.

On the other hand, the most propagated interpretation of the problem of time of the Wheeler-DeWitt equation (3.15) is that time must be reintroduced into the quantum theory by means of an auxiliary physical entity whose values can be correlated with the values of other physical entities. This correlation allows in principle to analyze the evolution of physical quantities with respect to the "auxiliary internal time". Since there is no clear definition of the auxiliary internal time, one can only use the imagination to choose a quantity as the time parameter. For instance, if we have a physical quantity which classically depends linearly on time, it could be a good candidate for an auxiliary internal time. Although the linearity seems to be a reasonable criterion, it is not a necessary condition. Examples of this type of auxiliary internal time are the very well analyzed minisuperspaces of quantum cosmology. In particular, one could select the auxiliary internal time as one of the scale factors of homogeneous cosmological models. The volume element which is a combination of scale factors would be also a good choice since in most cases it evolves in cosmic time and reproduces the main aspects of cosmological evolution. The volume element has also been used recently in loop quantum cosmology [18, 19]. Certain low energy limits in string theory contain a tachionic field which linearly evolves in time and, consequently, could be used as auxiliary internal time for quantization 20. We will consider these examples with some more details in section 6

Nevertheless, it is not clear at all if the procedure of fixing an auxiliary internal time can be performed in an exact manner and, if it can be done, whether the results of choosing different auxiliary times can be compared and are somehow related. Finally, a most controversial point is whether such an auxiliary time can be used to relate the usual concepts of spacetime.

In the last section we mentioned that the canonical quantization procedure implies that the fields to be quantized are defined on a background spacetime. In quantum field theory, the Minkowski spacetime with its set of preferred inertial frames plays the role of background spacetime. In general relativity there is no place for a background metric. In fact, the entries of the metric are the physical entities we need to quantize. This rises a new problem. If we success in quantizing the spacetime metric, we will obtain quantum fluctuations of the metric which make impossible the definition of spacelike, null or timelike intervals. But the starting 
commutation relations require the existence of a well-defined spacetime interval. For instance, the first commutation relation of Eq.(3.11) is usually interpreted as reflecting the fact that the points $\mathbf{x}$ and $\mathbf{x}^{\prime}$ are separated by a spacelike interval. However, there is no background metric to define this causal structure. Moreover, if we would choose an arbitrary background metric, the quantum fluctuations of that metric could completely change the causal character of the interval. So we are in a situation in which if we want to solve the original problem, we must violate one the most important postulates needed to find the solution. Obviously, this is not a good situation to begin with.

\section{Canonical quantization in minisuperspace}

The first attempt at minisuperspace quantization is due to DeWitt [21], although the concept of minisuperspace was introduced by Misner [22] some years later. At that time Wheeler [23] suggested the idea of superspace as the space of all threegeometries as the arena in which the geometrodynamics develops. A particular four-geometry being a trajectory in this space. Later, Misner applied the Hamiltonian formulation of general relativity to cosmological models, having in mind the quantization of these cosmological models. He introduced the concept of minisuperspace and minisuperspace quantization or quantum cosmology to describe the evolution of cosmological spacetimes as trajectories in the finite dimensional sector of the superspace related to the finite number of parameters, needed to describe the $t=$ const. slices of the models and the quantum version of such models, respectively.

In the early 70's the minisuperspace models and their quantum version were extensively studied, however, the interest in them decreased at the middle of this decade till Hartle and Hawking [24 revived the field in the early 80's emphasizing the path-integral approaches. This started a lively resurgence of interest in minisuperspace quantization.

In 1987 Macías, Obregón, and Ryan [25] introduced the supersymmetric quantum cosmology approach by applying $(N=1)$ supergravity to quantum minisuperspaces in order to obtain the square root of the Wheeler-DeWitt equation, which governs the evolution of the quantum cosmological models in the standard approach. In 1988 D'Eath and Hughes [26] constructed a locally supersymmetric 1-dimensional model for quantum cosmology, based on a particular case of the Friedmann-Robertson-Walker spacetime (see also [27]). Later on, these results where generalized to include Bianchi cosmological models, supersymmetric matter, and cosmological constant [28, 29, 30].

In 1994 Carrol, Freedman, Ortíz, and Page 33, showed that there is nophysical states in $N=1$ supergravity, unless there exist an infinite set of gravitino modes. In 1998 Macías, Mielke, and Socorro 31] showed that there are no-physical states in supersymmetric quantum cosmology. 
As stated in 34, one of the greatest difficulties with quantum cosmology has always been the seductive character of its results. It is obvious that taking the metric of a cosmological model, which is truncated by an enormous degree of imposed symmetry and simply plugging it into a quantization procedure cannot give an answer that can be in any way interpreted as a quantum gravity solution. What people do is to assume that one can represent the metric as a series expansion in space dependent modes, the cosmological model being the homogeneous mode, and that in some sense one can ignore the dependence of the state function on all inhomogeneous modes. This artificial freezing of modes before quantization represents an obvious violation of the uncertainty principle and cannot lead to an exact solution of the full theory. However, the results of applying this untenable quantization procedure have always seemed to predict such reasonable and internally consistent behavior of the universe that it has been difficult to believe that they have no physical content.

The minisuperspace is often known as the homogeneous cosmology sector, as mentioned above, infinitely many degrees of freedom are artificially frozen by symmetries. This reduction is so drastic that only an unphysical finite number of degrees of freedom is left. The requirement of homogeneity limits the allowed hypersurfaces to the leaves of a privileged foliation, which is labeled by a single "time" variable. One can parametrize such hypersurfaces of homogeneity by the standard Euler angles coordinates and characterize the spatial metric uniquely by three real parameters, $\Omega, \beta_{ \pm}$. The $\Omega$ is related to the volume of the hypersurface $\Sigma$ as follows:

$$
\Omega=\ln \int_{\Sigma} d^{3} x|q(x)|^{1 / 2} .
$$

The $\beta$ parameters describe the anisotropy of the hypersurface $\Sigma$. Due to the symmetry of the model, the super-momentum constraints are identically satisfied, while the super-Hamiltonian constraint reduces to:

$$
H_{\perp}=-p_{\Omega}^{2}+p_{+}^{2}+p_{-}^{2}+\exp (-\Omega)\left[V\left(\beta_{+}, \beta_{-}\right)-1\right]=0 .
$$

The potential $\left[V\left(\beta_{+}, \beta_{-}\right)-1\right]$ is a combination of exponential terms, it vanishes at the origin and it is positive outside of it 35 . The parameter $\Omega$ is usually considered as a kind of "auxiliary internal time" (see section 6). A systematic analysis of the global time problem for homogeneous cosmological models seems to lead, quite generally, to the lack of a global time function. Even the volume time $\Omega$ is not globally permitted, for instance in oscillating models, since the universe would attend a given value $\Omega<\Omega_{\max }$ at least twice, once when expanding, and once when recontracting.

Additionally, the Wheeler-DeWitt equation based on one particular choice of time variable, like $\Omega$ in this case, may give a different quantum theory than the same equation based on another choice of the time variable. This is what Kuchar called the multiple choice problem [5].

It is dangerous to draw conclusions from minisuperspace models to full quantum gravity. Minisuperspace spacetimes possess a privileged foliation by leaves of 
homogeneity which does not exist in a generic spacetime. Kuchař and Ryan 34 showed that even in the simple case of a microsuperpace (a reduced minisuperspace) the result of canonical quantization is not related to the quantization of the seed minisuperspace. One should try to avoid common practice, which consists of solving a time problem for a model way down in the hierarchy, and jumping to the conclusion that the time problems of quantum gravity are removed by the same treatment.

\section{Canonical quantization in midisuperspace}

The simplest generalization of the homogeneous models are the Gowdy cosmological models, since they possesses two Killing vectors and therefore two ignorable coordinates, reducing the problem to time (as in standard quantum cosmology) and one spacial coordinate, which completely eliminates homogeneity and leads to a system with an infinite number of degrees of freedom, i.e. a true field theory on a midisuperspace. Gowdy cosmologies are widely studied midisuperspace models.

Moreover, the canonical quantization of $N=1$ supergravity in the case of a midisuperspace described by Gowdy $T^{3}$ cosmological models has been already studied in [36]. The quantum constraints were analyzed and the wave function of the universe was derived explicitly. Unlike the minisuperspace case, it was shown that physical states in midisuperspace models do exist. The analysis of the wave function of the universe leads to the conclusion that the classical curvature singularity present in the evolution of Gowdy models is removed at the quantum level due to the presence of the Rarita-Schwinger field. Since this supegravity midisuperspace model shares the same problem as other midisuperspace models, which consists in the lacking of a well-defined time parameter, in this work a classical solution was used to drive the evolution in time.

The midisuperspace models provide a canonical description of Einstein spacetimes with a group of isometries. Symmetries remove infinitely many degrees of freedom of the gravitational field, but there remain still infinitely many degrees of freedom. In spite of this simplification, the midisuperspace constraints of general relativity are still complicated functionals of the canonical variables.

The study of midisuperspace models and covariant field systems, like string models, indicates that if there exists an auxiliary internal time which converts the old constraints of general relativity into a Schrödinger equation form, such a time variable is non-local functional of the geometric variables.

The Gowdy $T^{3}$ cosmological models have been analyzed in the context of non-perturbative canonical quantization of gravity [37, 38, 39]. The arbitrariness in the selection of a time parameter is a problem that immediately appears in the process of quantization. For a specific choice of time it was shown that there does not exist an unitary operator that could be used to generate the corresponding quantum evolution. Therefore, even in the case of midisuperspace models there is no natural time parameter. 


\section{The problem of time}

Quite a lot of different proposals have been made over the years on how to interpret time in quantum gravity, i.e., the time paradox. Kuchař [5] classified them in three basic approaches. It should be stressed that the boundaries of these interpretations are not clearly defined:

1. Internal Time. Time is hidden among the canonical variables and it should be identified prior to quantization. The basic equation upon the interpretation is based in a Schrödinger equation, not a Wheeler-DeWitt one. Nevertheless, this interpretation is susceptible to the multiple choice problem, i.e., the Schrödinger equation based on different time variables may give different quantizations.

(a) Matter clocks and reference fluids. The standard of time is provided by a matter system coupled to geometry, instead by the geometry itself. The intrinsic geometry and extrinsic curvature of a spacelike hypersurface enter into the constraints of general relativity in a very complicated way. Nothing in the structure of the mentioned constraints tell us how to distinguish the true dynamical degrees of freedom from the quantities which determine the hypersurface. The founding fathers of general relativity suggested a conceptual devise which leads exactly to that, i.e., the reference fluid. The particles of the fluid identify space points and clocks carried by them identify instants of time. This fixes the reference frame and the time foliation. In this frame and on the foliation, the metric rather than the geometry becomes measurable. The concept of reference fluid goes back to Einstein [40, and to Hilbert 41] who formalized the idea that the coordinate system should be realized by a realistic fluid carrying clocks which keep a causal time. They imposed a set of inequalities ensuring that the worldlines of the reference fluid be timelike and the leaves of the time foliation be spacelike.

The reference fluid is traditionally considered as a tenuous material system whose back reaction on the geometry can be neglected. There is just matter but not enough to disturb the geometry. Instead of deriving the motion of the fluid from its action, one encodes it in coordinate conditions. Those are statements on the metric which holds in the coordinate system of the fluid and are violated in any other coordinate system. Such standpoint makes difficult to consider the reference fluid as a physical object which in quantum gravity could assume the role of an apparatus for identifying spacetime events.

In order to turn the reference fluid into a physical system, it is possible to picture the fluid as a realistic material medium and devise a Lagrangian which describes its properties. By adding this Lagrangian to the Einstein-Hilbert Lagrangian, the fluid is coupled to gravity. Other possibility is to impose the coordinate condition before variation by 
adjoining them to the action via Lagrange multipliers. The additional terms in the action are parameterized and interpreted as matter source.

(b) Cosmological time. In one special case, the reference fluid associated with a coordinate condition allows a geometrical interpretation. This is the unimodular coordinate condition, i.e., $\left|g_{\mu \nu}\right|^{1 / 2}=1$, fixing the spacetime volume element. These unimodular coordinates were proposed by Einstein 42. By imposing the unimodular condition before rather than after variation, a law of gravitation with unspecified cosmological constant is obtained 43, reducing the reference fluid to a cosmological term. The cosmological constant appears as a canonical conjugate momentum to a time coordinate, i.e., the cosmological time.

The path-integral version of this approach has been used by Sorkin 44 45. to show that in a simple model of unimodular quantum cosmology the wave function remains regular as the radius of the universe approaches the classical singularity, but its evolution is non-unitary. Moreover, it has been shown by Heneaux and Teilteboim 46. that the increment of the cosmological time equals the four-volume enclosed between the initial and the final hypersurfaces.

Unruh and Wald [4] suggested that any reasonable quantum theory should contain a parameter, called Heraclitian time, whose role is to set the conditions for measuring quantum variables and to provide the temporal order of such measurements. The problem with this suggestion is that the cosmological time is not in any obvious way related with the standard concept of time in relativity theory. The basic canonical variables, the metric $q_{i j}$ and its conjugate momentum $\pi^{i j}$ are always imposed to be measured on a single spacelike hypersurface rather than at a single cosmological time. In order to be able to introduce a particular hypersurface, one needs to specify functions of three coordinates, instead of a single real parameter, i.e., the absolute time of Newtonian mechanics. Consequently, it remains the question in what sense the cosmological time sets the conditions of quantum measurements.

The cosmological time does not fix the conditions for a measurement uniquely, since it it cannot differentiate between the infinitely many possible hypersurfaces of the equivalence class, in order to know in which one the geometrical variables are to be measured. In other words, the hypersurfaces parametrized with different values of the cosmological time are allowed to intersect and cannot be causal ordered as the Heraclitian time requires. Therefore, cosmological time (Heneaux-Teilteboim volume) is not a functional time. Relativity time is a collection of all spacelike hypersurfaces and no single parameter is able to label uniquely so many events. 
(c) Time and tachyons. The specific form of the low energy action of the tachyon dynamics reads [20]:

$$
S=-\int d^{p+1} x V(T) \sqrt{1+\eta^{\mu \nu} \partial_{\mu} T \partial_{\nu} T},
$$

where $p=9$ for strings type IIA or IIB, and $p=25$ for bosonic strings, $\eta^{\mu \nu}=\operatorname{diag}(-1,1,1, \ldots, 1)$, and $V(T)$ is the potential of the tachyon $T$. Sen [20] proposed that, at the classical level, solutions of the equations of motion of the field theory described by (6.1), at "late time" are in one to one correspondence with configuration of non-rotating, non-interacting dust. At "late time" the classical vacuum solutions of the equations of motion approach $T=x^{0}=$ time coordinate, making $T$ a candidate for describing time at the classical level. On the other hand, at "late time" the quantum theory of the tachyon $T$ coupled to gravity leads to a Wheeler-DeWitt equation independent of $T$, whereas for "early time" or "finite time" the resulting Wheeler-DeWitt equation has a non-trivial dependence on $T$ in the considered region.

Nevertheless, it is well known that the classical tachyon dynamics, when quantized coupled to gravity, may not describe correctly the physics arising from the quantum string theory. Additionally, since the tachyon is identified with a configuration of non-rotating and incoherent dust, its role as time variable shares all the diseases, mentioned above, of reference fluids. Therefore, even in string theory the time paradox remains unsolved.

2. Wheeler-DeWitt framework. The constraints are imposed in the metric representation leading to a Wheeler-DeWitt equation. The dynamical interpretation asserts that the solutions would be insensitive to the time identification among the metric functions. This interpretation has to deal with the fact that the Wheeler-DeWitt equation presents familiar problems when one tries to turn the space of its solutions into a Hilbert space. Hence, the statistical interpretation of the theory is based on the inner product. Moreover, if there is a Killing vector, no energy operator commutes with the general relativity constraint $H_{\perp}$, and the construction of the Hilbert space fails. Even if there exists a timelike Killing vector, the positivity of the inner product requires that the potential in the super-Hamiltonian is non-negative.

The semiclassical interpretation hides the problem of time behind an approximation procedure. It claims that the Wheeler-DeWitt equation for a semiclassical state approximately reduces to the Schrödinger equation, and the Klein-Gordon norm reduces to the Schrödinger norm. Unfortunately, it achieves the positivity of the norm at an unacceptable price of suspending the superposition principle 490 .50. When the semiclassical interpretation is applied to quantum gravity properly the problem of separating the classical modes defining time from the quantum modes arises. In other words, this 
means that quantum gravity would have a probabilistic interpretation only if it is classical.

3. Quantum gravity without time. This interpretation claims that one does not need time to interpret quantum gravity and quantum mechanics in general. Time may emerge in particular situations, but even if it does not, quantum states still allow a probabilistic interpretation [15, 16, 17.

Its difficulty stands on the fact how to explain quantum dynamics in terms of constants of motion. The existing proposals are ambiguous, since the replacement of the classical global time parameter by an operator is ambiguous and its consequences lead to the multiple choice problem and to the problem of how to construct a Hilbert space [5].

As it is well known, in canonical formalism, gauge transformations are generated by constraints linear in the momenta, and they move a point in the phase space along, to what is usually called, an orbit of the gauge group. Moreover, two points on the same orbit are physically indistinguishable and represent two equivalent descriptions of same physical state. An observable should not depend on description of the chosen state, the state must be the same along the given orbit, i.e., its Poisson bracket with all the constraints must vanish.

On one hand, all the physical content of general relativity is contained in the constraints and the observables are those dynamical variables that have vanishing Poisson brackets with all constraints. In particular, due to the fact that the diffeormorphims constraint generates a gauge, i.e., the group of spatial coordinates diffeomorphisms. Therefore, any observable in general relativity must be invariant under diffeomorphisms.

On the other hand, the super-Hamiltonian constraint generates the dynamical change of the geometrical dynamical variables from one hypersurface to another, i.e., any dynamical variable which commutes with the super-Hamiltonian must be the same on every hypersurface and it must be constant of motion. Nevertheless, in order to be able to maintain that the quantum observables are those which commute with all the constraints of general relativity seems to imply that our quantum universe can never change. The transformations generated by the super-Hamiltonian should not be interpreted as gauge transformations. Two points on the same orbit of the super-Hamiltonian transformations are two events in the dynamical evolution of the system which are distinguishable instead of been two descriptions of the same physical state.

Second quantization. There exists a belief that the second quantization solves the problem of time in quantum theory of a relativistic particle. The second quantization approach to quantum field theory is based on the construction of a Fock space, i.e., one takes a one-particle Hilbert space $F_{(1)}$. From the direct product of the one-particle states, the states which span the $\mathrm{N}$-particle sector $F_{(N)}$ are constructed. The Fock space $F$ is then the direct sum of all such sectors, i.e., $F=F_{(0)} \oplus F_{(1)} \oplus F_{(2)} \oplus \cdots$, where $F_{(0)}$ is spanned 
by the vacuum state. It is clear that the Fock space $F$ can be defined only if the one-particle state $F_{(1)}$ is a Hilbert space. This brings us to the Hilbert space problem for a relativistic particle. The absence of a privileged oneparticle Hilbert space structure is the source of ambiguities in constructing a unique quantum field theory on a dynamical background [5].

A closer look to the second quantization approach reveals that it does not really solve the problem of time evolution and its formalism resists an operational interpretation, like the problems presented by the indefinite inner product of the Klein-Gordon interpretation, which are faced by suggesting that the solutions of the Wheeler-DeWitt equation are to be turned to operator. This is analogous to subjecting the relativistic particle, whose state is described by the Klein-Gordon equation, to second quantization.

In full, the second quantization merely shifts the problem of time to a different level without really solving it.

\section{Conclusions}

Since the concepts of time in quantum mechanics and general relativity are drastically different from each other, generalizations of the usual quantum theory are required to deal with quantum spacetime and quantum cosmology [51]. That is due to the fact that the usual framework for quantum theory assumes a fixed background spacetime geometry. Physical states are defined on spacelike hypersurfaces in this geometry and evolve unitarily in between such hypersurfaces in the absence of measurements and by state vector reduction on them when a measurement occurs. The inner product is defined by integrals over fields on a spacelike hypersurface. Nevertheless, at the quantum realm, spacetime geometry is not fixed, but a dynamical variable fluctuating and without definite value. It is not possible to determine whether two given nearby points on a spacetime manifold are spacelike separated or not. Instead, the amplitudes for predictions are sums over different metrics on the manifold. Additionally, points separated by a spacelike intervals in one metric could be timelike separated in another metric, that contributes just as significantly to the sum. Moreover, quantum theory does not provide a natural time parameter and the quantum constraints of general relativity do not contain any time parameter. For this reason, standard quantum mechanics needs to be generalized to accommodate quantum spacetime.

On the other hand, the application of quantum mechanics to quantum cosmology also requires another kind of generalization of the standard formulation. Standard quantum mechanics predicts the outcome of measurements carried out on a system by another system outside it. However, in cosmology there is no outside. Therefore, quantum cosmology requires a quantum mechanics for closed systems, i.e., a generalization of the standard quantum theory.

All the attempts to implement the canonical quantization procedure to quantize systems in which time is not Newtonian do not provide a reasonable description 
of the corresponding quantum system. The quantization of general relativity has been an open problem for more than 70 years and the leading present approaches, string theory and loop quantum gravity, are far from providing an ultimate solution, although many technical problems have been attacked and partially solved in the past 20 years. Nevertheless, it seems that the main conceptual problems, especially the one related to time, are still not well understood. In our opinion, it is not possible to reconciliate and integrate into a common scheme the absolute and non-dynamical character of Newtonian time of canonical quantization with the relativistic and dynamical character of time in general relativity. What is needed is a radical change of perspective either in general relativity or in quantum mechanics. That is to say, we need either a theory of gravity with an non-dynamical Newtonian time or a quantum theory with a dynamical time in its construction. We believe that what requires radical changes is the canonical quantization procedure in such a way that the concept of time enters it in a more flexible manner. The issue of time remains as an open problem.

\section{References}

[1] G. Amelino-Camelia, C. Lämmerzahl, A. Macías, and H. Müller: "The Search for Quantum Gravity Signals", in: Gravitation and Cosmology, eds. A. Macías, C. Lämmerzahl, and D. Núñez (American Institute of Physics, 2005) pp. 30-80.

[2] C. Kiefer, Quantum Gravity (Clarendon Press, Oxford, 2004).

[3] H.D. Zeh, The physical basis of the direction of time (Springer-Verlag, Berlin, 2001).

[4] J.J. Halliwell, Derivation of the Wheeler-DeWitt equation from a path integral for minisuperspace models, Phys. Rev. D38 (1988), 2468.

[5] K. Kuchař, "Time and interpretation of quantum gravity", in: Proc. 4th Canadian Conf. on General Relativity and Relativistic Astrophysics, eds. G. Kunstatter, D. Vincent, and J. Williams (World Scientific, Singapore, 1992).

[6] N. M. J. Woodhouse, Geometric Quantization (Clarendon Press, Oxford, 1980).

[7] C. J. Isham, "Topological and global aspects of quantum theory", in: Relativity, Groups and Topology II: Les Houches 1983, eds. B. S. DeWitt and R. Stora (North-Holland, Amsterdam, 1984) pp. 1059.

[8] A. Ashtekar, Lectures on Non-Perturbative Canonical Gravity (World Scientific, Singapore, 1991).

[9] R. Wald, Quantum Field Theory in Curved Spacetime and Black Hole Thermodynamics (University of Chicago Press, Chicago, 1994).

[10] R. Arnowitt, S. Deser, and C.W. Misner, "The dynamics of general relativity", in: Gravitation: An introduction to current research. ed. L. Witten (Wiley, New York, 1962).

[11] J. W. York, Role of conformal three-geometry in the dynamics of gravitation, Phys. Rev. Lett. 28 (1972) 1082.

[12] B.S. DeWitt, "The space-time approach to quantum field theory", in: Relativity, groups, and topology II eds. B.S. DeWitt and R. Stora (North-Holland, Amsterdam, 1984) pp. 381. 
[13] J. Butterfield and C. J. Isham, Spacetime and philosophical challenge of quantum gravity, arXiv: qr-qc/9903072 Ch. 4.5

[14] S. Carlip, Quantum Gravity: a progress report, Rep. Prog. Phys. 64 (2001) 885.

[15] C. Rovelli, Quantum mechanics: A model, Phys. Rev. D42 (1990) 2638.

[16] C. Rovelli, Time in quantum gravity: An hypotesis, Phys. Rev. D43 (1991) 442.

[17] C. Rovelli, Quantum Gravity (Cambridge University Press, Cambridge, UK, 2004).

[18] M. Bojowald, Loop quantum gravity: II. Volume operators, Class. Quantum Grav. 17 (2000) 1509.

[19] M. Bojowald, Loop quantum gravity: II. Discrete time evolution, Class. Quantum Grav. 18 (2001) 1071.

[20] A. Sen, Time and Tachyon, Int. J. Mod. Phys. A18 (2003) 4869.

[21] B.S. DeWitt, Quantum Theory of Gravity I. The canonical theory, Phys. Rev. 160 (1967) 1113.

[22] C.W. Misner, "Minisuperspace", in: Magic without Magic: John Archibald Wheeler, ed. J. R. Klauder (Freeman, San Francisco, 1972) pp. 441.

[23] J.A. Wheeler, "Geometrodynamics and the issue of the final state" in: Relativity, Groups and Topology, ed. C. DeWitt and B. DeWitt. (Gordon and Breach, New York, 1964), pp. 459 ff.

[24] J. B. Hartle and S.W. Hawking, Wave function of the universe, Phys. Rev. D28 (1983) 2690.

[25] A. Macías, O. Obregón and M. P. Ryan, Quantum Cosmology: The Supersymmetric Square Root, Class. Quantum Grav. 4 (1987) 1477.

[26] P.D. D'Eath, D.I. Hughes, Supersymmetric minisuperspace, Phys. Lett. B214 (1988) 498.

[27] P.D. D'Eath, D.I. Hughes, Minisuperspace with local supersymmetry, Nucl. Phys. B378 (1992) 381.

[28] P. D. D'Eath, Quantization of the supersymmetric Bianchi I model with a cosmological constant, Phys. Lett. B320 (1994) 12.

[29] A.D.Y. Cheng, P.D. D'Eath, and P.R.L.V. Moniz, Quantization of the Bianchi IX model in supergravity with a cosmological constant, Phys. Rev. D49 (1994) 5246.

[30] A.D.Y. Cheng, P.D. D'Eath, and P.R.L.V. Moniz, Quantization of a FriedmannRobertson-Walker model in $N=1$ supergravity with gauged supermatter, Class. Quantum Grav. 12 (1995) 1343.

[31] A. Macías, E.W. Mielke, and J. Socorro, Supersymmetric quantum cosmology: the physical states, Phys. Rev. D57 (1998) 1027.

[32] A. Macías, E.W. Mielke, and J. Socorro, Supersymmetric quantum cosmology for Bianchi class A models, Int. J. Mod. Phys. D7 (1998) 701.

[33] S.M. Carroll, D. Z. Freedman, M.E. Ortiz, and D. Page,Physical states in canonically quantized supergravity, Nucl. Phys. B423 (1994) 661.

[34] K. V. Kuchař and M. P. Ryan, Is minisuperspace quantization valid? Taub and Mixmaster, Phys. Rev. D40 (1989) 3982.

[35] M.P. Ryan, Hamiltonian Cosmology (Springer-Verlag, New York, 1972); M.P. Ryan and L.C. Shepley: Homogeneous Relativistic Cosmologies (Princeton University Press, New Jersey, 1975). 
[36] A. Macías, H. Quevedo and A. Sánchez, Gowdy $T^{3}$ Cosmological Models in $N=1$ Supergravity, arXiv: grqc/0505013.

[37] A. Ashtekar and M. Pierri, Probing quantum gravity through exactly soluble midisuperspaces, J. Math. Phys. 37 (1996) 6250.

[38] G. A. Mena-Marugan, Canonical quantization of the Gowdy model, Phys. Rev. D56 (1997) 908.

[39] A. Corichi, J. Cortez, and H. Quevedo, On unitary time evolution in Gowdy $T^{3}$ models, Int. J. Mod. Phys. D11 (2002) 1451.

[40] A. Einstein, "Uber die spezielle und allgemeine Relativitätstheorie (Vieweg, Braunschweig, 1920).

[41] D. Hilbert, Grundlagen der Physik, 2Mitt., Nachr.Eiss.Göttinger 53 (1917).

[42] A. Einstein, Die Grundlagen der allgemeinen Relativitätstheorie, Ann. Phys. (Leipzig), 49 (1916) 769.

[43] W.G. Unruh, Unimodular Theory of Canonical Quantum Gravity, Phys. Rev. D40 (1989) 1048.

[44] R. Sorkin, On the role of time in the sum over histories framework for gravity, Int. J. Theor. Phys. 33 (1994) 523.

[45] R. Sorkin, Forks on the road, on the way to quantum gravity, Int. J. Theor. Phys. 36 (1997) 2759.

[46] M. Henneaux and C. Teitelboim, The Cosmological Constant and General Covariance, Phys. Lett. B222 (1989) 195.

[47] W.G. Unruh and R.M. Wald, Time and the interpretation of canonical quantum gravity, Phys. Rev. D40 (1989) 2598.

[48] K. Kuchař, Does an Unspecified Cosmological Constant Solve the Problem of Time in Quantum Gravity? Phys. Rev. D43 (1991) 3332.

[49] D.N. Page, Density matrix of the universe, Phys. Rev. D34 (1986) 2267.

[50] D.N. Page and W.K. Wootters, Evolution without evolution: Dynamics decribed by stationary observables, Phys Rev. D27 (1983) 2885.

[51] J.B. Hartle, General Relativity and Quantum Mechanics, Int. J. Mod. Phys. A16 (2001) 1 .

\title{
Acknowledgment
}

One of us (A.M.) thanks Claus Lämmerzahl and Bertfried Fauser for enlightening discussions in Bremen and Blaubeuren, respectively. This work was supported by CONACyT grants 48601-F, and 48404-F.

\author{
Alfredo Macías \\ Departamento de Física, \\ Universidad Autonoma Metropolitana-Iztapalapa, \\ A.P. 55-534, México D.F. 09340, México \\ e-mail: amac@xanum.uam.mx
}


Hernando Quevedo

Instituto de Ciencias Nucleares, Universidad Nacional Autónoma de México

A.P. 70-543, México D.F. 04510, México

e-mail: quevedo@nuclecu. unam.mx 